\title{
LA MARCACIÓN DEL GÉNERO EN LOS SUSTANTIVOS DEL ESPAÑOL: ENTRE LA FLEXIÓN Y LA DERIVACIÓN
}

\author{
Jorge E. Murillo
}

\begin{abstract}
RESUMEN
En las siguientes reflexiones, se esquematizan las posiciones referentes al tema de la categoría del género y se busca encontrar un planteamiento uniforme que permita visualizar, desde un ángulo más global, los procesos morfológicos que entran en juego en la marcación del género del nombre sustantivo.
\end{abstract}

\begin{abstract}
The following reflections outline the points of view regarding gender category, and seek to find an uniform statement that may allow to visualize, from a more global angle, the morphological processes that are at work for marking the gender of the noun.
\end{abstract}

A pesar de la gran cantidad de material bibliográfico referente al tema de la categoría género de los sustantivos del español, muchas dudas persisten aún sobre si estos morfemas, como veremos bastante variados, pertenecen a la categoría del paradigma flexivo o el derivativo.

Tradicionalmente, las gramáticas prescriptivas no encuentran problemas para considerar el morfema de género como parte de la flexión, pero recientes estudios, principalmente fundamentados en teorías morfológicas modernas, cuestionan este hecho. No se ha llegado todavía a postular una teoría totalmente completa al respecto; lo que sí se tiene como cierto es el hecho de que, a la luz de nuevas perspectivas, no se pueden aceptar a ciegas los datos de las gramáticas tradicionales.

En las siguientes reflexiones, se esquematizan las posiciones mencionadas y se busca encontrar un planteamiento uniforme que permita visualizar, desde un ángulo más global, los procesos morfológicos que entran en juego en la marcación del género del nombre sustantivo. 


\section{La gramática prescriptiva}

En esencia, los postulados de la gramática prescriptiva, aquella que da las reglas para el uso de la lengua, se resumen en el texto clásico Esbozo de una nueva gramática para la lengua española, de la Real Academia de la Lengua (1974). Otros gramáticos importantes, cuya posición a este respecto sin embargo no dista mucho de la académica, son Andrés Bello, Rafael Seco y últimamente Emilio Alarcos Llorach, entre otros. Se esbozan, a continuación, los lineamientos generales que guían este tipo de estudios.

Tradicionalmente, se reconoce que existen en español solamente dos géneros: el masculino y el femenino. Como sucedió con todas las lenguas romances, los sustantivos neutros latinos desaparecieron como tales en su paso del latín vulgar a las lenguas romances, distribuyéndose en las dos categorías resultantes.

En el Esbozo (1974: 172-3) se dice que: "Por el género, los nombres sustantivos se dividen en español en femeninos y masculinos. La categoría nominạl del neutro no existe en la lengua española. Decimos que un nombre es femenino o masculino cuando las formas respectivamente femeninas o masculinas del artículo y de algunos pronombres, caracterizadas las primeras por el morfema de género -a, y las segundas por el morfema de género -o, -e o por ningún morfema, se agrupan directamente o aluden a él fuera de esta construcción."

Como se aprecia, la definición tiene carácter sintáctico (es decir, se define el género del sustantivo a partir de su correlación con otros lexemas de la frase) y taxonómico, pues se asignan a cada género morfemas determinados.

Por otra parte, se reconoce la identificación del género gramatical con el sexo del ente designado en aquellos casos en que se hace referencia a personas o animales: "Si los nombres apelativos sustantivos son de persona, tenemos la idea de que femeninos y masculinos, tal como acabamos de definirlos, designan respectivamente mujer y varón, o hembra y macho de algunas especies animales". La excepción a tal principio estaría dada por el sincretismo presente en las formas de masculino plural, pues pueden hacer referencia a un grupo donde existen elementos de cada uno de los sexos: por ejemplo, hijos o hermanos, pueden designar grupos heterogéneos en cuanto al sexo. A este respecto, Alarcos (1994: 62-3) plantea que: “...de los dos géneros, el masculino es el de mayor extensión, y el femenino el de mayor intensión. Quiere decir esto que cuando el uso lingüístico ha decidido la indistinción de los géneros, lo que se emplea en la expresión es el significante masculino. Así en los padres, los reyes, los hombres se significa la fusión de ambos géneros (esto es, el padre y la madre, el rey y la reina, los hombres y las mujeres) cuando tales expresiones se oponen a otras como los hijos, los príncipes, los animales; pero sigue vigente el masculino cuando se manifiestan en oposición a los femeninos las madres, las reinas, las mujeres."

Los procedimientos lingüísticos más comunes para expresar la diferencia de sexo en los nombres son dos:

1. La moción: cambio o alargamiento de la terminación para expresar el femenino:

$\begin{array}{ll}\text { gat-o } & \text { gat-a } \\ \text { gall-o } & \text { gall-ina } \\ \text { héro-e } & \text { hero-ína } \\ \text { actor } & \text { actr-iz }\end{array}$



abad
abad-esa
sacerdot-e
sacedot-isa

2. La heteronimia: uso de palabras o raíces diferentes:

$\begin{array}{ll}\text { caballo } & \text { yegua } \\ \text { hombre } & \text { mujer } \\ \text { yerno } & \text { nuera } \\ \text { padre } & \text { madre }\end{array}$

En otros casos, a pesar de que existe diferencia biológica en cuanto al sexo, gramaticalmente no queda evidenciada la variación; este es el caso de los nombres llamados comunes, en los que la única forma de distinción es la sintáctica, es decir, agregando los artículos 0 adjetivos respectivos:
el turista la turista
el periodista la periodista
el comediante la comediante
el testigo la testigo
el guía la guía
el soprano la soprano

Es de interés destacar, en cuanto a estos sustantivos, que a pesar de que la gramática prescriptiva asigna femenino a muchos nombres de oficio o profesión mediante el procedimiento de moción (juez>jueza; presidente $>$ presidenta $>$; médico $>$ médica o jefe $>$ jefa), en el habla corriente predomina la idea de que son comunes y de allí las denominaciones 'la médico' o 'la juez'. Probablemente, esto tenga su explicación en un fenómeno social evidente estas profesiones o oficios históricamente han sido ocupadas por seres de sexo masculino y por ello, cuando sobreviene la igualdad real de la mujer, en tiempos modernos, en la lengua persiten aún resabios de la predominancia de uno de los sexos.

En cuanto a los animales, en pocos casos se usa la moción o la heteronimia como mecanismos lingüísticos para marcar la diferencia sexual; más bien, la mayoría de las veces debe acudirse a procedimientos léxicos (agregar un lexema aclaratorio) para hacer la distinción, dado que ni el mismo artículo funciona como elemento discriminatorio. A estos nombres tradicionalmente se les conoce como epicenos.
la culebra (hembra) la culebra (macho)
el pez (hembra) el pez (macho)
la paloma (hembra) la paloma (macho)
el cocodrilo (hembra) el cocodrilo (macho)

En el caso de que se trate de seres sin sexo biológico determinado, la asignación del género posee carácter arbitrario, es decir, que esos referentes no tienen ninguna característica "biológica" que los haga pertenecer a una u otra categoría. Así 'mesa' o 'lápiz', son sustantivos cuyo género solo podría explicarse a la luz del desarrollo histórico de la lengua. 
En este caso, las terminaciones varían en su rango y en muchas ocasiones una misma terminación puede aparecer en sustantivos de distinto género: el mal, la sal; el sol, la col; el lapiz, la cerviz, etc. Sin embargo, según la Academia, es importante el dato de que la mayoría de los nombres de cosa en -o son masculinos, a excepción de algunos que han conservado su género originario: la mano, por ejemplo. En otros casos, la incongruencia se explicaría por reducción del vocablo: la moto (la motocicleta), la polio (la poliomielitis) o la foto (la fotografía). De igual manera, son masculinos aquellos terminados en los siguientes sufijos:

1. -ete: brazalete, sorbete, banquete.

2. -ón (diminutivos derivados de sustantivos): notición, perezón, mujerón 1 .

3. -or: colador, tractor, cursor.

4. - -il (acentuado): redil, atril, proyectil.

También pertenecen a este género, algunos nombres terminados en -a y que provienen del griego: anatema, tema, lema, problema, dilema, etc.

Por su parte, ciertos sufijos se asocian con el género femenino:

1. -triz: cicatriz, bisectriz.

2. -cie: calvicie, molicie.

3. -ez/eza: estupidez, delgadez, escasez, pobreza, tristeza, belleza.

4. - -(i)dad: bondad, verdad, brutalidad.

5. - -ión: inversión, diversión, división.

6. -tud: plenitud, multitud, virtud.

7. -umbre: pesadumbre, lumbre, costumbre.

En cuanto a las series léxicas o grupos semánticos, los nombres que las integran adquieren el género del término que las domina; así, los ríos, montes, volcanes u otros accidentes geográficos, llevan el mismo género que el sustantivo genéricos que los designa: el Nilo, el Amazona, el Fuji, el Titicaca (el lago). Los colores, sería otro caso: el (color) lila, el escarlata, el azul, el rojo.

Para terminar con este recuento, se hará referencia a nombres tradicionalmente conocidos como ambiguos, que con una misma forma adoptan los dos géneros. Los hay de dos tipos:

1. Aquellos que varían parcialmente su significado al adoptar uno u otro género:

el cometa la cometa

el frente la frente

el orden la orden

el lente la lente

el Génesis la génesis

2. Aquellos que no varían semánticamente ${ }^{2}$ :

el / la azúcar

el/la calor

el/la mar 
Queda de esta manera establecida, grosso modo, la taxonomía manejada por la gramática prescriptiva, la que se enseña normalmente en el sistema escolar. Definitvamente, aún desde una óptica bastante general, esta taxonomía presenta bastantes problemas, basta con pensar en algunos contraejemplos, lo cual no es una tarea muy difícil, tal y como quedará demostrado en la reseña de las posiciones más modernas al respecto.

\section{El modelo estructuralista}

La corriente estructuralista norteamericana intentó por su parte dar una explicación convincente al complicado problema de la marcación del género en los sustantivos españoles. Utilizando las técnicas típicas de segmentación y clasificación, algunos estructuralistas como Philip W. Klein (1989), siguiendo a Sol Saporta (1962), identifica los siguientes morfemas básicos, con sus respectivos alomorfos:

1. Masculinos: $\{-\mathrm{o}\},\{-\mathrm{e}\}$ y $\{\varnothing\}$, para los casos hermano, padre y señor, respectivamente.

2. Femeninos: $\{-\mathrm{a}\},\{-\mathrm{e}\}$ y $\{\varnothing\}$, para los vocablos hermana, madre y mujer, respectivamente.

Para Klein (1989), esta clasificación presenta problemas por sí misma pues hay categorías cruzadas como $\{-\mathrm{e}\}$ y $\{-\varnothing\}$ : "Unfortunately, these results are not entirely satisfactory: Saporta himself is disturbed that they include 'contrastive' forms which are in fact identical in the case of both -e and $\varnothing$. That is, the masculine -e and its femenine counterpart are homophonous, and the analysis countenances two $\varnothing$ allomorphs in opposition to each other." Además, para este lingüista el panorama se complica por el hecho de que otras vocales pueden funcionar también como morfemas de género indistintamente para femenino o masculino: $\{-\mathrm{u}\}$, como en 'espíritu' o 'tribu'; $\{$-a $\}$ también para masculino, como en 'día' y $\{-o\}$ para femenino, como en "mano". Por ello, señala: "We are now faced not only with indistinguishable -e, $-\varnothing$, but also with homophonous - - , -a (supposedly the gender vowels par excellence), as well as with 'opposed' -us. Thus, these two gender morphemes turn out to have identical allomorphs, which reduces the claim that they contrast in the speech stream to near vacuity."

La distribución de estos morfemas y sus alomorfos, sin embargo, es aclarada por Anderson (1961), quien propone que el morfema es $\{-0\}$ con los alomorfos [-o ], [-e ] y [ - $]$ ]. Para él, "That / -e / and / $-\varnothing /$ are allomorphs of the morpheme [(o)] is illustrated in still another way. In addition to paradigmatic opposition with [(a)], c.f., / xéf-e /, / xéf-a / and / francés- $\varnothing$ /, / francés-a /, the relationship is shown by recourse to the basic allomorph /-o / which is always found added to the dimunitive or augmentative suffix. Thus / -o / alternates syntagmatically with the non-basic allomorph, e.g., / xéf-e /, / xef-esít-o /; / francés- $\varnothing$ /, / frances-ít-o /. Observation of roots such as / xéf- / and / francés-/ when follow by a dimunitive or augmentative reveals that even with these stems $[(0)]$ is represented by / -o / as well as by / -e / or / $\varnothing /$. On this basis, we consider /-o/ to be the basic allomorph."

No obstante, como más tarde lo analizaría el mismo Klein, no se puede pensar en -e como alomorfo de género, sino más bien como una vocal epentética que aparecería cuando la raíz termina en grupo consonántico o en una obstruyente simple que no sea / d / o / s /: 
"I have argued elsewhere that there are compelling reasons for not taking -e to be a gender allomorph at all: it has no consistent relationship to one gender over another and merits no morphemic status. It is rather an epenthetic vowel supplied by a purely phonological rule, merely to facilitate pronunciation..." Lo anterior tiene ya su fundamento teórico en la teoría generativa, la cual abordaremos más adelante.

Volviendo a la clasificación de Anderson para completar la visión estructural, él propone que los sustantivos pueden tener dos tipos de morfemas de género: los inflexivos y los derivativos. Los primeros estarían constituidos por las vocales señaladas, mientras que los segundos corresponderían a aquellos casos de moción en que, además del morfema inflexivo, existe otro derivativo entre este y la raíz; tal es el caso de nombres como:

$\begin{array}{ll}\text { cond-e } & \text { cond-es-a } \\ \text { gall-o } & \text { gall-in-a } \\ \text { sacerdot-e } & \text { sacerdot-is-a } \\ \text { poet-a } & \text { poet-is-a }\end{array}$

en donde se señalan con negrita los correspondientes sufijos derivativos.

\section{El análisis generativo}

Con la aparición de las teorías generativas de los años sesenta, los análisis realizados desde la lingǘstica estructural se ven ampliados con nuevas aclaraciones y premisas. Inclusive, lingüistas como los mencionados en el aparte anterior hacen referencias a puntos de vista generativos con el objeto de dar una visión más completa de sus propuestas.

El presupuesto del cual parten estos análisis es el de que los fenómenos derivativos deben ser analizados como parte del lexicón, en tanto que los inflexivos pertenecen al subcomponente fonológico. Además, la ventaja de este enfoque estriba en que, al contrario del estructuralista, se trabaja no solo con rasgos de superficie (el componente fonológico) sino que también se tiene en cuenta la estructura profunda y las variaciones entre una y otra serían explicadas a partir de reglas de generación.

En relación con la marcación del género, los generativistas consideran que es procedimiento gramatical que no pertenece a la morfología flexiva, sino más bien a la derivativa y que, por lo tanto, como afirma Cressy (1978): "All Spanish nouns are marked for gender in the lexicon. In some instances, the gender correlates with the semantic category of sex: hijo (masculine gender) 'son', hija (femenine gender) 'daughter'. In most cases, however, the gender is an arbitrary morphological feature devoid of any semantic content: caso (masculine gender) 'case', casa (femenine gender) 'house'."

Las premisas fundamentales que se aplican al respecto de la marcación genérica son las siguientes: en caso de que los sustantivos tengan género evidentemente marcado, aparecería en la superficie una -o para el masculino y una -a para el femenino. Si no lo tienen, estas palabras terminarían, fonéticamente, en una consonante o en una / -e /. Las excepciones las resume Cressey tal como sigue: "In the following examples, the articles ( $e l$ for masculine and $l a$ for femenine) are the only overt markings of the gender: el hombre feliz 'the happy man', la noche triste 
'the sad night', la virtud 'virtue', el ataúd 'the coffin'. There are also some outright exceptions which must be marked in the lexicon, such as: la mano 'the hand' and el poema 'the poem'."

De esta manera, en el caso de palabras como 'ataúd' y 'virtud' se propone una -e epentética en el nivel fonético, que aparecería en otros casos como el plural: 'ataúdes' y 'virtudes'. De acuerdo con esto, pues, las terminaciones de género de todos los sutantivos caerían en cualesquiera de las siguientes categorías: los que tienen género especificado: -o ó -a y los que terminan en -e, los cuales requerirían una especificación en el lexicón, de modo que al surgir en la superficie los artículos o adjetivos que los acompañan puedan ser generados con el género correspondiente. Lo anterior lo resume Cressey afirmando que: "...in the lexicon, all nouns are marked with respect to two features: [+/- masculine] and [+/- overt gender]; adjectives are all marked in the lexicon with respect to the feature [+/- overt gender] and acquire a specification for the feature [masculine] by a transformational rule which copies this feature specification from the noun onto the adjective."

Siguiendo con la descripción, afirma que tanto sustantivos como adjetivos carecen ¿u vocal final en el lexicón, pues estas son generadas luego como reflejo automático de las características mencionadas en el párrafo anterior. Por esto, un sustantivo tendría dentro del nivel léxico la siguiente subcategorización:

$$
\begin{array}{ll}
/ \mathrm{kas} / & {[+ \text { noun, }- \text { masculine, }, \text { overt gender }]} \\
/ \mathrm{ombr} / & {[+ \text { noun },+ \text { masculine, } \text {-overt gender }]}
\end{array}
$$

De esta manera, se explica la asignación del género en las primeras visiones generativistas que parten de Sol Saporta (1962) pasan por Cressy (1978) hasta llegar a Klein (1989). Sin embargo, también algunos lingüistas hispanos se han referido al asunto desde una posición generativa. D'Introno y otros (1988) resumen así lo hasta ahora establecido: "El clasificar como flexionales los fenómenos de género y número, tal como hace la tradición, oscurece el hecho de que en los sustantivos dichos fenómenos son independientes de la sintaxis, mientras que en los modificadores de los mismos sustantivos se trata de hechos sintácticos. En otras palabras, el género y número de los modificadores de un sustantivo $\mathrm{N}$ están determinados por el género y el número de $\mathrm{N}$, pero el género y el número de $\mathrm{N}$ no están determinados por los de ninguna palabra con la que $\mathrm{N}$ entra en relación sintáctica. Estos hechos se caracterizan en nuestro modelo haciendo que la formación del plural y la marcación de género, como reglas de formación léxica, se apliquen a los NN en el módulo léxico, pero a los modificadores (determinantes y adjetivos) en el módulo posléxico."

Queda así delimitada la descripción generativa que se hace de los procesos de asignación del género para los sutantivos. A su vez, lo anterior también justifica el hecho de que hayamos tomado solo al sustantivo como base para el presente estudio, pues los otros elementos nominales, a saber, adjetivos y artículos, son componentes del módulo posléxico sintáctico, y en esa medida la asignación de los morfemas de género dependen del nombre al cual se refieren.

Resta ahora, sin embargo, hacer una valoración de conjunto de todas las propuestas hasta ahora descritas que nos conduzca, por otra parte, a encontrar un común denominador con el objeto de comprender mejor este proceso morfológico. 


\section{Hacia una visión de conjunto}

Diacrónicamente, la visión de la gramática prescriptiva es importante, en tanto nos proporciona las bases para acercamientos posteriores. Indudablemente, el fundamento del prescriptivismo está dado por la influencia de la gramática latina, que sirvió como modelo ya a los primeros gramáticos de la nueva lengua castellana, como es el caso de Nebrija.

El latín, como sabemos, tenía tres géneros: el masculino, el femenino y el neutro. En el desarrollo de las distintas lenguas romances el neutro se perdió y quedaron como diferenciados solo masculino y femenino. Todos los sustantivos neutros tuvieron que readecuarse a los nuevos procesos morfológicos del castellano; algunos, como los terminados en -um (vinum $>$ vino ) o en -us (pectus $>$ pecho) pasaron al masculino y otros, unos pocos, atraídos por la -a femenina pasaron con este género chusma, calma ${ }^{3}$. Lo anterior, sumado al hecho de que los sustantivos de la tercera, cuarta y quinta declinaciones tuvieron también que adecuarse a los nuevos géneros existentes, dio en español una variada gama de posibilidades de desinencias en los nombres. No nos interesa destacar aquí todos los cambios surgidos en esta reorganización, que bien resume Cano Aguilar (1988) afirmando que: "Por tanto, la historia del 'género' en castellano antiguo, y a lo largo de todo el español, se limita, aparte de la conservación etimológica del género latino, mayoritaria, a los cambios de género para adecuarlo a la desinencia (o al significado), a los cambios de terminación para adecuarla al género, y en ocasiones a ambos fenómenos a la vez".

Lo que sí resulta importante de destacar es el hecho de que todas estas readecuaciones son la causa directa de la falta de unificación, en cuanto a terminaciones se refiere, en nuestra lengua actual. Y, por otra parte, que la gramática prescriptiva siempre ha tratado de describir el sistema de desinencias de género con arreglo a la evolución histórica. Baste, para comprobar lo anterior, hojear cualquier estudio en este sentido, en los cuales se alude frecuentemente a la etimología de las palabras con el objetivo de justificar su desinencia o, en su defecto, notar la falta de motivación para el cambio.

Las desviaciones de las "supuestas" desinencias que deberían presentar los sustantivos de acuerdo con su género han sido una preocupación constante de los estudiosos del español. Todas estas llamadas anomalías han dado lugar a ulteriores intentos de clasificación o aclaración; tal es el caso de Angel Rosenblat (1962), quien describe minuciosamente todos aquellos casos de sustantivos que se apartan de la norma en la construcción del género, para concluir que: "Tenemos, pues, en castellano un sistema de terminaciones -o, -a que responden a la oposición de m. y f. Pero dentro de él tenemos otro, de órbita menos amplia, de masculinos en -a. Estos masculinos ejercen también atracción analógica aún la anomalía se acrecienta analógicamente".

Por otra parte, las clasificaciones prescriptivas, en vista de lo anterior, no siguen criterios unitarios para el estudio del género: en algunos casos, se basan en lo morfológico (morfemas $-0,-a$ ), en otros, en lo sintáctico (determinación del género por medio del artículo en los llamados comunes) y en otros, criterios léxicos (marcación por medio de palabras "macho' y 'hembra' en los epicenos). Otro desacierto importante de esta visión, según nuestro criterio, es la búsqueda de una motivación entre sexo/género, en vista de que parten del presupuesto de que existe una motivación natural entre ambas categorías dentro de la lengua, lo cual ha quedado demostrado como falso por los estudios posteriores. Esto se explica porque 
existe una mayoría de sustantivos en los que la diferencia de género pareciera estar motivada por la diferencia de sexo del referente (sobre todo en los animados), lo cual ha provocado una tendencia a tratar de explicar todo el sistema con arreglo a este tipo de sustantivos. De ahí todas las tendencias a etiquetar como anómalos los nombres que no corresponden con los parámetros anteriores, cuando en realidad lo que se da es que la marcación del género de los sustantivos españoles es arbitraria, es decir que las categorías sexo/género no están necesariamente correlacionadas.

Otro de los problemas de la gramática tradicional es su marcado interés en agrupar las dos clases más importantes de sustantivos (animados e inanimados) de acuerdo con los mismos parámetros, con el consecuente resultado de un panorama donde, en lugar de la simetría, priva la anomalía.

En ese sentido, los nuevos estudios descriptivistas (el del estructuralismo y el de la gramática generativa) parten del claro principio de que existen esas dos clases de sustantivos y de que la marcación del género en cada una de ellas responde a lineamientos un tanto difeientes. De hecho, la mayoría de estos estudios se centra en los primeros, los animados, en vista de que en ellos existe mayor regularidad en los morfemas asociados con el género; no así en los inanimados, en cuyo caso no hay asociación de ningún tipo, es decir, que el género no tiene ninguna correlación con el sexo o la forma del objeto ${ }^{4}$.

El estructuralismo logra delimitar, aunque no en forma precisa, los morfemas asociados con el rasgo en estudio. Los generativistas, partiendo de esa base, proponen reglas que aclaran aún más estos procesos fonológicos. Sin embargo, estas mismas reglas no dan cuenta absoluta de todas las posibilidades y, por ende, terminan acudiendo a la excepción para explicar los casos anómalos. La conclusión a la que llega Klein evidencia lo anterior: "The "gender vowel' analysis developed here so far is more restrictive than its structuralist forebearer. It includes only -o and -a in that category, redefined as "vowel typically associated with a certain gender', rather than 'morpheme expressing a particular gender'; the grammar links no other vowel to either gender. Final -e regularly appears epenthetically, for phonological reasons, on nouns of both gender, and a very small number of nouns end, unexpectedly, in other vowels $(u, i)$ or else end in o although they are femenine, or in a even though they are masculine."

El principal aporte del generativismo es que plantea cómo el género de los sutantivos es parte del lexicón, y no de la sintaxis, y en esa medida, a pesar de que existen ciertas vocales asociadas con el femenino o el masculino, no se podría establecer una asociación definitiva y estable. Si se parte del principio de que el género de los nombres está asignado en el componente léxico, o sea que es derivativo y no flexivo, es bastante fácil entonces explicar el comportamiento de esta categoría gramatical.

Ante este panorama, incompleto aún según nuestro criterio, proponemos una hipótesis general, que como tal necesitaría de corroboración, máxime de estudios socio y psicolingüísticos. Existen dos procedimientos para la marcación del género de los sutantivos del español: los animados tienden a una regularización del paradigma, con una clara orientación a buscar una asociación con el género natural del elemento designado. En este caso, las vocales -o y -a corresponderían al masculino y al femenino respectivamente. No se contarían los casos de heteronimia (hombre-mujer) sino solo los de moción, ni aquellos pocos casos en los que, por tratarse de profesiones asociadas generalmente al sexo masculino, la forma femenina no se ha usado, como albañil, plomero y otras. Las anomalías dentro de este grupo 
(los llamados nombre comunes y los epicenos) creemos que han venido en un proceso de regularización o de búsqueda de la simetría . De ahí que encontremos, en el habla popular, casos como testigo/testiga, modisto/modista, comediante/comedianta o miembro/miembra. Evidente también es el hecho de que algunos otros se resisten a este cambio: periodista, comunista, artista, no sin haber oído, por lo menos en mi experiencia de habla, casos en los que se hace la diferencia.

Respecto de los tradicionalmente llamados epicenos, apoya nuestra hipótesis el que los nombres de animales más comunes a la vida doméstica tienen forma diferenciada: perro/perra, gato/gata, gallo/gallina, mulo/mula, caballo/yegua, toro/vaca. Los menos cercanos a nuestra experiencia no tienen diferenciación genérica asociada con el sexo natural, pero encontramos en la mayoría de los casos, una tendencia a ello: la pareja rata/ratón se entiende como correlacionada (aunque sabemos que son dos especies diferentes), al igual que abeja/abejón u hormiga/hormigón. Definitivamente, el desinterés del hablante por establecer la diferencia de sexo (o la imposibilidad de reconocerlo a simple vista) explicarían por qué hay unos pocos casos en los que no se da la diferencia: insecto, culebra, o la mayoría de nombres de aves.

En cuanto a los objetos inanimados, el género sería arbitrario, es decir, sin relación alguna con la forma del objeto. Para estos, el género estaría dado, en términos de la gramática generativa, en el lexicón y por lo tanto sería derivativo. Para los animados, planteamos que el género tiende hacia la motivación y, por lo tanto, estaría dado en el módulo sintáctico y correspondería más a un proceso de flexión.

\section{Notas}

1. Nótese lo interesante del hecho de que, a pesar de que se derivan de nombres femeninos, estas formas cambian su género al agregar la terminación -ón, aún en el caso de mujer, en el que supuestamente debería guardarse la correlación género/sexo.

2. En este caso, más bien, la variación se justifica por razones sociolingüísticas, pues el uso de uno u otro género depende del registro o contexto en que se use. Así, 'la mar' es expresión poética o de gentes de origen rural.

3. Me parece importante aquí destacar el criterio de Andrés Bello (edición de 1988) quien considera que: "Atendiendo a la construcción del adjetivo con el sustantivo, no hay nada más que dos géneros en castellano, masculino y femenino; pero atendiendo a la representación o reproducción de ideas precedentes por medio de los demostrativos, hay tres géneros masculino, femenino y neutro [...].

Primeramente, los demostrativos sustantivos se representan unos a otros. Si digo, por ejemplo, "Eso me agrada", no puedo añadir, "Es preciso no pensar más en él", ni "en ella", sino "en ello". Así eso, masculino en cuanto pide la terminación masculina del adjetivo que lo modifica ( eso es bueno, eso es falso ), no es masculino ni femenino en cuanto a su reproducción o representación en el razonamiento; y por consiguiente es neutro bajo ese respecto, porque neutro quiere decir ni uno ni otro, esto es, ni masculino ni femenino."

4. Hay casos de objetos inanimados donde pareciera haber alguna regularidad en la diferencia de género y la forma del objeto. Por lo menos, en el español de Costa Rica, existe la pareja 'mango/manga' para referirse a la fruta. El masculino designa una variedad alargada mientras que la segunda, una redonda. 


\section{Bibliografía}

Alarcos Llorach, Emilio. 1994. Gramática de la lengua española. Madrid: Espasa Calpe.

Anderson, James M. 1991. "The morphophonemics of gender in Spanish nouns". Lingua. 10 (3): 285-96.

Bello, Andrés. 1988. Gramática de la Lengua Castellana. Madrid: Ed. Arco Libros S.A.

Cano Aguilar, Rafael. 1988. El español a través de los tiempos. Madrid: Ed. Arco Libros S.A.

Cressy, William W. 1978. Spanish Phonology and Morphology: A generativ view. Washington D.C.: Georgetown University Press.

D’Introno, Francesco et al. 1988. Fundamentos de Lingüística Hispánica. España: Editorial Playor.

García-Page, Mario. 1991. "Un aspecto de morfología flexiva del español actual”. Español actual. (56): 23-8.

Harris, James W. 1991. "The exponence of gender in Spanish". Linguistic Inquiry. 1 (22): 2762.

Klein, Phillip W. 1989. "Spanish 'gender' vowels and lexical representation”. Hspanic Linguistics. 3 (1-2): 147-62.

Menéndez Pidal, Ramón. 1987. Manual de Gramática Histórica Española. Madrid: Ed. Espasa Calpe.

Nissen, Uwe K. 1986. "Sex and gender specification in Spanish". Journal of pragmatics. (10): 285-96.

Romero Gualda, María Victoria. 1989. El nombre: sustantivo y adjetivo. Madrid: Ed. Arco Libros S.A.

Rosenblat, Ángel. 1962. "Morfología del género". Nueva Revista de Filología Hispánica. 16 (84): 31-80.

Real Academia de la Lengua Española. 1973. Esbozo de una nueva gramática de la lengua española. Madrid: Espasa Calpe.

Seco, Manuel. 1972. Gramática esencial del español. Madrid: España Ed. Aguilar. 
